\title{
The Exotic Characteristics of Centauro-I - A model to describe Centauro-I
}

\author{
A. Ohsawa ${ }^{a}$, E.H. Shibuya ${ }^{b}$ and M. Tamada ${ }^{c}$ \\ (a) Institute for Cosmic Ray Research, University of Tokyo, Kashiwa, 277-8582 Japan \\ (b) Instituto de Fisica, Universidade Estadual de Campinas, 13081 Campinas, Saõ Paulo, Brazil \\ (b) School of Science and Engineering, Kinki University, Higashi-Osaka, 577-8502, Japan \\ Presenter: M.Tamada (tamada@ele.kindai.ac.jp), jap-tamada-M-abs1-he21-oral
}

The features of the exotic cosmic-ray event "Centauro-I" are: (1) 60 - 90 hadrons, unaccompanied by $\gamma$-rays, incident upon the chamber, (2) a reasonable lateral spread of these hadrons, and (3) no nuclear interactions of these hadrons in the upper chamber but $20-30$ interactions in the target layer. We propose a model of strange quark matter among the primary cosmic rays to describe the event.

\section{Introduction}

The exotic event Centauro-I consists of several tens of visible showers of energies above $1 \mathrm{TeV}$ in the lower chamber of the two-storey structure. In our previous paper [1] we have shown, after detailed re-examination of the event, that Centauro-I is produced by a bundle of hadrons (more than $28 \mathrm{C}$-jets in quantity), incident upon the upper chamber, with a lateral spread. There is, however, no (or only one) shower in the upper chamber, which means that these hadrons are not accompanied by any $\gamma$-rays and that the hadrons do not interact in the upper chamber. The first point is quite exotic for ordinary high energy cosmic-ray events in the atmosphere. That is, ordinary high energy cosmic-ray events generally consist more of $\gamma$-rays and less of hadrons when they are detected by emulsion chambers. The second point is also exotic because these hadrons cause nuclear collisions in the target layer, a $23 \mathrm{~cm}$ thickness of petroleum pitch equivalent to $0.46 \lambda$ ( $\lambda:$ the mean free path of nucleons), while they do not in the upper chamber, a $7.8 \mathrm{~cm}$ thickness of Pb equivalent to $0.49 \lambda$. Fig. 1 shows an illustration of the characteristics of the Centauro-I. The thickness of the chamber materials are tabulated in Table 1. In this paper, we propose a model to describe these embarrassing features of Centauro-I.

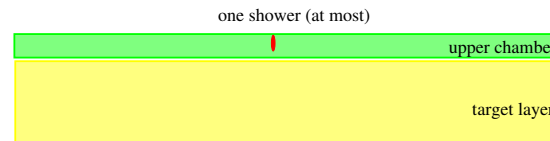

air gap

138 showers $(E>0.2 \mathrm{TeV})$ with spread about $1 \mathrm{~cm}$

\begin{tabular}{|c|c|c|}
\hline I. & 11 & lower chamber \\
\hline 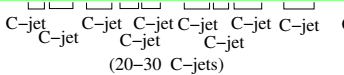 & C-jet & \\
\hline
\end{tabular}

Figure 1. Illustration of the characteristics of Centauro-I. Two-storey chamber consists of the upper chamber, the target layer, air gap and the lower chamber. No (or one at least) shower is observed in the upper chamber but many (20 - 30 ) $\mathrm{C}$-jets are observed in the lower chamber.

\section{Number of hadrons incident upon the upper chamber}

The energy spectrum of showers (138 showers of $E_{\gamma} \geq 0.2 \mathrm{TeV}$ ) observed in the lower chamber are well reproduced by a superposition of $20-30 \mathrm{C}$-jets, i.e., hadron collisions in the target layer. Assuming that the 
Table 1. Thickness of material in Chamber 15

\begin{tabular}{||l|c|c|c||}
\hline \hline & Thickness & Effective thickness & Collision Prob. \\
\hline \hline Upper chamber & $1.3 \times 6 \mathrm{~cm} \mathrm{~Pb}(0.49 \lambda)$ & $t_{1}=0.33 \lambda$ & 0.28 \\
\hline Target layer & $23 \mathrm{~cm} \mathrm{Pitch}(0.46 \lambda)$ & $t_{2}=0.62 \lambda$ & 0.33 \\
\hline Lower chamber & $1.0 \times 8 \mathrm{~cm} \mathrm{~Pb}(0.5 \lambda)$ & $t_{3}=0.31 \lambda$ & 0.10 \\
\hline
\end{tabular}

${ }^{*}$ Conditions for shower detection are taken into account. $\lambda$ is the collision mean free path of nucleons.

hadrons which initiate the $\mathrm{C}$-jets are nucleons, the number of nucleons incident upon the upper chamber can be estimated to be $(20-30) / 0.33 \simeq 60-90$ where the factor of 0.33 is the interaction probability of nucleons in the target layer (see Table 1). In this estimation, and hereafter, we assume for simplicity that all the collisions are visible. If $60-90$ nucleons are incident upon the upper chamber, the number of nucleons which induce $\mathrm{Pb}$-jets in the upper chamber is $(60-90) \times 0.28=17-25$ where the figure 0.28 is the interaction probability of nucleons in the upper chamber (see Table 1). However we observe, at most, only one shower in the upper chamber. It is impossible to attribute this large discrepancy to a fluctuation of interaction probabilities, and we need some physical mechanism to describe the discrepancy.

In summary, we have to describe the quite exotic features.

1. The hadrons, incident upon the upper chamber, are not accompanied by $\gamma$-rays.

2. The hadrons do not cause collisions (or do not cause collisions of visible energy) in the upper chamber, while they cause $20-30$ collisions in the target layer.

\section{A Model to describe Centauro-I}

Bjorken and McLerran proposed a model [2] for Centauro when Centauro-I was initially reported [3]. It assumed a glob of quark matter in a meta-stable state among the primary cosmic rays. They suggested that the glob in meta-stable state heats up while passing through the atmosphere and explodes into a number of hadrons without the emission of $\gamma$-rays. Note that the glob in this model does not emit any (or an appreciable number of) high energy particle(s) while passing through the atmosphere before its explosion. Another explanation of the Centauro is also proposed by Ref.[4] assuming centauro fire-ball as SQM (Strange Quark Matter). Centauro fire-ball penetrate deep in the atmosphere and decay into non-strange baryons and strangelet(s). Adopting these ideas, we assume that a large SQM entered the atmosphere and fragmented into a bundle of small strangelets in the atmosphere without the emission of $\gamma$-rays (see Fig. 2). These small strangelets are incident upon the upper chamber and explode into nucleons in the upper chamber ${ }^{1}$.

Let us assume that a bundle of small strangelets (with multiplicity $N$ ) is produced just above the chamber ${ }^{2}$ A small strangelet explodes into $n$ nucleons either in the upper chamber or in the target layer without emission of $\gamma$-rays. We assume that the explosion length of small strangelets is $L$ in $\mathrm{Pb}$ and the collision mean free path of nucleons is $\lambda(=16.0 \mathrm{~cm} \mathrm{~Pb})$. The nucleons which interact in the upper chamber initiate $\mathrm{Pb}$-jets in the upper chamber. One can see that the model reduces the number of collisions in the upper chamber by adjusting the value for explosion length $L$ of the small strangelets. Fig. 3 shows that the number ratio between nucleon collisions in the target layer, $I_{2}$, and in the upper chamber, $I_{1}$, depends weakly on the explosion length

\footnotetext{
${ }^{1} \mathrm{We}$ assume in the present paper for simplicity that the hadrons produced in the explosion are nucleons. If we assume pions for these hadrons, we have to assume a further mechanism to exclude $\pi^{0}$ 's among the pions in order for the scenario below to hold.

${ }^{2}$ Therefore we can neglect the thickness of the atmosphere which the bundle passes through. (If the bundle is produced high above the chamber, some of the small strangelets would explode into nucleons in the atmosphere according to the explosion length.)
} 


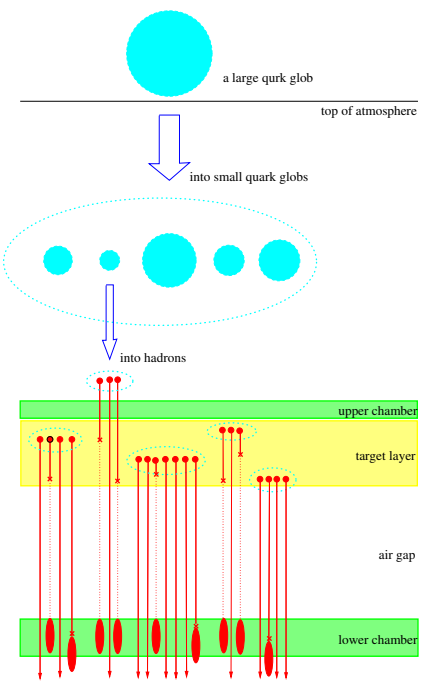

Figure 2. Illustration of fragmentation of a large strange quark glob into small strangelets in the atmosphere (with multiplicity $N$ ), and explosions of the respective small strangelets into hadrons (with multiplicity $n$ ) either in the atmosphere or in the chamber. The produced hadrons (solid lines) cause nuclear collisions (crosses) in the upper chamber, in the target layer or in the lower chamber, or leave the lower chamber (arrows) without collisions. The $\gamma$-rays among the produced particles in respective nuclear collisions originate a diffused cascade shower, a bundle of cascade showers or a cascade shower, respectively, in the lower chamber, each of which is drawn by an ellipse.

$L$ of the small strangelets, increasing gradually. The values are tabulated in Table 2 , where the numbers in the fifth row are the expected number of collisions in the upper chamber when $20-30 \mathrm{C}$-jets are observed in the lower chamber. The last row is the total number of nucleons after all the small strangelets have exploded into nucleons, or the equivalent mass number of the large strangelet.

Table 2. Ratio of the number of collisions in the upper chamber and in the target layer

\begin{tabular}{||l|c|c|c|c||}
\hline \hline Explosion length $L$ & $0.5 \lambda$ & $1.0 \lambda$ & $2.0 \lambda$ & $3.0 \lambda$ \\
\hline \hline$I_{1}$ & $7.90 \times 10^{-2}$ & $4.41 \times 10^{-2}$ & $2.31 \times 10^{-2}$ & $1.57 \times 10^{-2}$ \\
\hline$I_{2}$ & $2.73 \times 10^{-1}$ & $1.85 \times 10^{-1}$ & $1.08 \times 10^{-1}$ & $7.63 \times 10^{-1}$ \\
\hline$I_{2} / I_{1}$ & 3.46 & 4.18 & 4.68 & 4.86 \\
\hline Expected number of collisions & \multicolumn{4}{||}{} \\
\hline in the upper chamber & $5.8-8.7$ & $4.8-7.2$ & $4.3-6.4$ & $4.1-6.2$ \\
\hline in the target layer & $20-30$ & $20-30$ & $20-30$ & $20-30$ \\
\hline Number of nucleons $n N$ & $74-110$ & $108-162$ & $184-270$ & $262-303$ \\
\hline \hline
\end{tabular}

If we take $L \geq 2 \lambda$ for the explosion length of the small strangelets, the expected number of collisions in the upper chamber is $4.3-6.4$ and we can describe why there is at most one shower in the upper chamber, taking into account the fluctuation of collision probability. We assume tentatively that the explosion length is $2 \lambda$ for the small strangelets, i.e., $L=2.0 \lambda=32.0 \mathrm{~cm} \mathrm{~Pb}=362 \mathrm{~g} / \mathrm{cm}^{2}$. Then estimated number of nucleons after the explosion of all the strangelets is $n N=184-270$.

\section{Discussions}

The exotic characteristics of Centauro-I are

1. $60-90$ hadrons, without accompaniment of $\gamma$-rays, incident upon the upper chamber.

2. At most one nuclear collision of these hadrons in the upper chamber but $20-30$ collisions in the target layer 


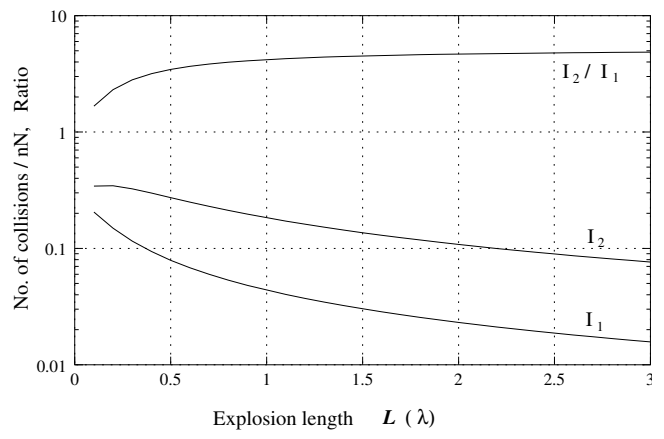

Figure 3. Expected number (divided by $n N$ ) of collisions in the upper chamber, $I_{1}$, and that in the target layer, $I_{2}$, when $N$ small strangelets enter the chamber and explode into $n$ nucleons respectively with explosion length $L$. The ratio between $I_{2}$ and $I_{1}$ is shown together. One can see that the number ratio increases gradually with increasing explosion length $L$.

To describe Centauro-I we assume a glob of strange quark matter in a meta-stable state among the primary cosmic rays, after Bjorken and McLerran. The glob heats up during its passage through the atmosphere and fragments into a bundle of small strangelets without the emission of any $\gamma$-rays. In the case of Centauro-I the fragmentation occurred just above the detector, and the bundle of small strangelets hit the upper chamber directly. The small strangelets heat up passing through the chamber materials to explode into nucleons either in the upper chamber or in the target layer with the explosion length $L=2 \lambda=32.0 \mathrm{~cm} \mathrm{~Pb}$. (We assume that the produced hadrons are nucleons for the sake of simplicity.) Then the expected number of collisions in the upper chamber is $4.3-6.4$ for the number of observed C-jets, $20-30$. It is not a rare fluctuation that there is no (or only one) collision for an expected $4.3-6.4$ collisions in the upper chamber. One can see that present model also describes the absence of $\gamma$-rays in the upper chamber (the point 1, above), too.

According to our model for Centauro-I, there exist globs of strange quark matter among the primary cosmic rays. These globs, equivalent to $180-270$ nucleons, pass through a large thickness of material, i.e., a half of the atmosphere of the earth, without producing an appreciable number of particles.

There are reports of similar type of exotic events, which may be described by strange quark matter among the primary cosmic rays, by balloon experiments. Two events with expected atomic number $Z \sim 14$ and mass number $A \sim 370$, are pointed out as being possibly strange quark matter by the authors [5]. The other event is an extremely penetrating (more than $200 \mathrm{~g} / \mathrm{cm}^{2}$ ) track. The estimated energy and the mass number of the event are $5.4 \times 10^{2} \mathrm{GeV}$ and 64 , respectively. [6].

It is interesting to see that the intensity of ultra high energy air showers (UHEASs) exceeding GZK cut-off energy, detected by AGASA experiment[7], is consistent with an extrapolation of these intensities of balloon events and Centauro-I, though the energy estimation of UHEASs should be examined carefully.

\section{References}

[1] A. Ohsawa, E.H. Shibuya, and M. Tamada, Phys. Rev. D 70, 074028 (2004)

[2] J.D. Bjorken and L.D. McLerran, Phys. Rev. D 20, 2353 (1979)

[3] C.M.G. Lattes, Y. Fujimoto, and S. Hasegawa, Phys. Rep. 65, 151 (1980); M.Tamada, Nuovo Cimento 41B, 245 (1977)

[4] A.D. Panagiotou, A. Petridis and M. Vassiliou, Phys. Rev. D 45, 3134 (1992); A.L.S.Angelis and A.D.Panagiotou, G. Phys. G 23, 2069 (1997); E.Gladysz-Dziadus and Z. Wlodarczyk, G. Phys. G 23, 2057 (1997)

[5] T. Saito, Y. Hatano, Y. Fukada, and H. Oda, Phys. Rev. Lett. 65, 2094 (1990)

[6] M. Ichimura et al., Nuovo Cim. 106 A, N.6, 843 (1993)

[7] M. Takeda et al., Astropart. Phys. 19447 (2003). 